\title{
Resveratrol attenuates endothelial oxidative injury by inducing autophagy via the activation of transcription factor EB
}

\author{
Xi Zhou, Jining Yang, Min Zhou, Yu Zhang, Yang Liu, Pengfei Hou, Xianglong Zeng, Long Yi and Mantian Mi
}

\begin{abstract}
Background: Endothelial oxidative injury is a key event in the pathogenesis of atherosclerosis (AS). Resveratrol (RSV) attenuates the oxidative injury in human umbilical vein endothelial cells (HUVECs). Autophagy is critical for the RSVinduced protective effects. However, the exact underlying mechanisms haven't been completely elucidated. Thus, we aimed to explore the role of autophagy of the anti-oxidation of RSV and the underlying mechanism in palmitic acid (PA)-stimulated HUVECs.

Methods: HUVECs were pretreated with $10 \mu \mathrm{M}$ of RSV for $2 \mathrm{~h}$ and treated with $200 \mu \mathrm{M}$ of PA for an additional $24 \mathrm{~h}$. Cell viability, intracellular reactive oxygen species (ROS) and malondialdehyde (MDA) levels were estimated with a microplate reader and confocal microscope. Autophagosomes were analyzed by transmission electron microscopy, while lysosomes by confocal microscopy. The expression of transcription factor EB (TFEB) and related genes were quantified by qRT-PCR assay. Furthermore, TFEB levels, autophagy, and lysosomes were examined by western blot assay.
\end{abstract}

Results: RSV pretreatment suppressed the PA-induced decline in cell viability and elevation in ROS and MDA levels in HUVECS. RSV pretreatment also increased LC3 production and P62 degradation while promoted the autophagosomes formation. However, 3-methyladenine (3-MA) treatment attenuated RSV-induced autophagy. RSV pretreatment upregulated the TFEB and TFEB-modulated downstream genes expression in a concentrationdependent manner. Additionally, in cells transfected with TFEB small interfering RNA, RSV-induced TFEB expression and subsequent autophagy were abolished. Meanwhile, the TFEB-modulated genes expression, the lysosomes formation and the RSV-induced anti-oxidation were suppressed.

Conclusions: In HUVECS, RSV attenuates endothelial oxidative injury by inducing autophagy in a TFEB-dependent manner.

Keywords: Resveratrol, TFEB, Autophagy, Endothelial cells, Atherosclerosis

\section{Background}

Atherosclerosis (AS) is the most serious threat to human cardiovascular health because it can lead to the coronary artery and cerebrovascular diseases, which is associated with a heavy economic burden $[1,2]$. As a multifactorial disease, AS is induced by several cardiovascular risk factors, especially the dysfunction of vascular endothelial cells $[3,4]$. Endothelial oxidative injury, induced by

\footnotetext{
* Correspondence: mi_mt2009@hotmail.com

Research Center for Nutrition and Food Safety, Chongqing Key Laboratory of Nutrition and Food Safety, Institute of Military Preventive Medicine, Third Military Medical University (Army Medical University), NO.30 Gao Tan Yan Street, Shapingba District, Chongqing 400038, People's Republic of China
}

palmitic acid (PA), which promotes apoptosis and causes endothelial dysfunction, is a driving force in the initiation and development of AS [5, 6]. Thus, attenuating endothelial oxidative injury is the key to reversing the progression of AS.

Resveratrol (3, 4', 5-trihydroxystilbene; RSV) is a naturally occurring polyphenolic compound that is mostly extracted from grapes and has been associated with multiple health benefits, such as anti-AS effects. According to the classic French paradox, one of the important reasons for the low incidence of cardiovascular diseases in France is that the French drink wine, in which RSV is

(C) The Author(s). 2019 Open Access This article is distributed under the terms of the Creative Commons Attribution 4.0 International License (http://creativecommons.org/licenses/by/4.0/), which permits unrestricted use, distribution, and 
the most effective antioxidant phytochemical [7]. We previously proved that RSV can attenuate endothelial dysfunction owing to its anti-oxidative bioactivity [8]. Nevertheless, the underlying mechanisms have not been fully clarified. Autophagy reportedly plays a key role in lipid catabolism and cellular clearance and affects various physiological processes [9-13]. Specifically, multiple research models have suggested that autophagy is critical for the RSV-induced protective effects [14, 15]. Thus, we wondered whether autophagy plays a key role in the RSV-induced anti-oxidative effects in human umbilical vein endothelial cells (HUVECs).

Although autophagy in response to metabolic cues has been studied for decades, the exact mechanisms mediating the autophagy induced by RSV and the related essential transcriptional regulation remain uncharacterized. Recent studies revealed that transcription factor EB (TFEB), which is a dominant regulator of the autophagylysosome pathway, also regulates organismal transcription and metabolism [16-18]. Moreover, increased TFEB activation may regulate autophagy induction and lysosomal biogenesis under starvation conditions, similar to the effects of caloric restriction. Additionally, TFEB activation may extend the lifespan of $C$. elegans, implying that increased longevity is regulated by nutrients [16]. A previous study showed that RSV can mimic the effects of caloric restriction and promote longevity [19]. Therefore, we hypothesized that the protective effect of RSV in response to endothelial oxidative injury occurs via TFEB-mediated autophagy. To prove this hypothesis, we studied the potential role of an RSV pretreatment in preventing PA-induced oxidative injury and in subsequently activating autophagy in HUVECs. Furthermore, we explored the expression of TFEB and downstream genes related to autophagy and lysosomal biogenesis, such as those encoding ATPase $\mathrm{H}+$ transporting V0 subunit D1 (ATP6VOD1), lysosomal-associated membrane protein 1 (LAMP-1), cathepsin B (CTSB), microtubule-associated proteins $1 \mathrm{~A} / 1 \mathrm{~B}$ light chain $3 \mathrm{~B}(M A P 1 L C 3 B)$, and $\mathrm{UV}$ resistance-associated gene (UVRAG). Additionally, based on a TFEB small interfering RNA (siRNA) transfection experiment, we investigated the role of TFEB in RSVinduced autophagy. This study demonstrated, for the first time, that RSV protects endothelial cells from oxidative injury by inducing autophagy in HUVECs, at least partially in a TFEB-dependent manner.

\section{Methods}

\section{Chemicals and reagents}

The culture medium of HyQ M199/EBSS (SH30351.01) and fetal bovine serum (SH30370.03) were purchased from HyClone Laboratories (Logan, UT, USA). Transresveratrol, dimethyl sulfoxide, PA, phosphate-buffered saline (PBS), 3-methyladenine(3-MA), the antibody of
Histone H3 and LC3 were obtained from Sigma-Aldrich (St. Louis, MO, USA). A Cell Counting Kit (CCK-8; CK04) was purchased from Dojindo Laboratories (Dojindo, Kumamoto, Japan). Reactive Oxygen Species (ROS) Assay Kit, Lipid Peroxidation malondialdehyde (MDA) Assay Kit, Lyso-Tracker Red and Hanks' Balanced Salt Solution (with $\mathrm{Ca}^{2+} \& \mathrm{Mg}^{2+}$ ) were obtained from the Beyotime Institute of Biotechnology. HRPconjugated anti-mouse and anti-rabbit secondary antibodies were purchased from Invitrogen. Antibodies of P62, TEFB, and LAMP1 were obtained from Cell Signaling Technology, whereas, $\beta$-actin antibody, fluorescein isothiocyanate (FITC)-conjugated secondary antibody, TFEB siRNA and control siRNA were purchased from Santa Cruz Biotechnology, Inc. (Santa Cruz, CA, USA).

\section{Cell culture and treatment}

According to the previous study, we isolated HUVECs from umbilical cord veins [8] and cultured with M199 medium added with $10 \%$ fetal bovine serum and $1 \%$ penicillin-streptomycin, at $37{ }^{\circ} \mathrm{C}$ and $5 \% \mathrm{CO}_{2}$. Cells from 3 to 6 passages were adopted to the following experiments. During the logarithmic growth phase, cells were pretreated with $10 \mu \mathrm{M}$ of RSV for $2 \mathrm{~h}$ and then exposed to $200 \mu \mathrm{M}$ of PA for an additional $24 \mathrm{~h}$. Cells were also exposed with 3-MA $(5 \mathrm{mM})$ for $1 \mathrm{~h}$ after the adding resveratrol for $2 \mathrm{~h}$. This study was approved by the ethics committee of Army Medical University and consent was attained by all the involved patients.

\section{Cell proliferation}

Cell proliferation was analyzed using a Cell Counting Kit-8 (Dojindo, Kumamoto, Japan) as previously described [20]. Briefly, PA was dissolved in $0.1 \mathrm{M} \mathrm{NaOH}$ at $70{ }^{\circ} \mathrm{C}$ to form a $100 \mathrm{mM}$ PA solution firstly. Then, we dissolve it in $10 \%$ BSA solution and stirred it in a $55^{\circ} \mathrm{C}$ water bath for about $3 \mathrm{~h}$. $0.22 \mu \mathrm{m}$ filter was used to sterilize the PA solution and save at $-20^{\circ} \mathrm{C}$ to prepare a PA reserve liquid. Then, 8000 cells were seeded into 96well microplates and then exposed to a series of PA concentrations $(0,100,150,200,250$ and $300 \mu \mathrm{M})$ for a series of time points (12, 16, 20 and $24 \mathrm{~h}$ ). To detect the effect of RSV on PA-induced oxidative stress damage, cells were treated with various concentrations of RSV $(0$, $0.1,1$ and $10 \mu \mathrm{M}$ ) for $2 \mathrm{~h}$, and subsequently exposed to $200 \mu \mathrm{M}$ of PA for an additional $24 \mathrm{~h}$. Next, $20 \mu \mathrm{L}$ of CCK- 8 solution was added to each well followed by $1-2$ $\mathrm{h}$ incubation at $37^{\circ} \mathrm{C}$. A monochromator microplate reader was used to measure the absorbance (Molecular Devices, Sunnyvale, CA, USA) at $450 \mathrm{~nm}$. Cell proliferation was calculated from the ratio of the optical density of the experimental cells to that of the control cells (set as $100 \%)$. 


\section{Western blot analysis}

The total cell lysate was analyzed by western blot analysis. Briefly, Equal amounts $(50 \mu \mathrm{g})$ of proteins were resolved by $10 \%$ sodium dodecyl sulfate-polyacrylamide gel electrophoresis and the electroblotted onto polyvinylidene difluoride membranes for western blot analysis. Blots were probed with primary antibodies overnight at $4{ }^{\circ} \mathrm{C}$. The primary antibodies are listed in Table 1 . Specially, the nuclear protein and cytoplasmic protein were isolated respectively by Nuclear and Cytoplasmic Protein Extraction Kit (P0027, Beyotime, China) according to the instruction. The two fractions of proteins were tested by western blot to quantify the protein level of nuclear TFEB and cytosolic TFEB. The protein level ratio of nuclear TFEB to cytosolic TFEB was calculated by which the value of nuclear TFEB/Histone $\mathrm{H} 3$ divided by cytosolic $\mathrm{TFEB} / \beta$-actin. Immune complexes were visualized using Immobilon Western Chemiluminescent HRP Substrate (Millipore, USA) and the signal was captured by Fusion FX (Vilber Lourmat, France). Densitometry analysis was computed using Image J software (NIH, MD, USA).

\section{RNA extraction and quantitative real-time polymerase chain reaction (qRT-PCR)}

RNAiso Plus reagent (Takara Bio, Japan) was used to harvest total RNA according to the manufacturer's instructions. qRT-PCR and data collection were performed by the qTower 2.2 real-time PCR system (Anakytik Jena, Germany) using SYBR Premix Ex Taq II (Tli RNaseH Plus) (Takara Bio, Japan). The primers for the targeted genes were produced by Sangon Biotech (Shanghai, China). Further, indicated genes were amplified according to the primers (Table 2). Relative foldchanges in gene expression were normalized to $\beta$-actin and analyzed by the $2^{-\Delta \Delta \mathrm{Ct}}$ method.

\section{siRNA assay}

The siRNA for TFEB (human, sc-38,509), control siRNA (sc-44,230) and siRNA Transfection Reagent (sc-29,528) were obtained from Santa Cruz Biotechnology (USA). $100 \mathrm{nM}$ of siRNA was applied to transfect HUVECs for 5-7 h, using Lipofectamine 2000 reagent (Invitrogen) following the manufacturer's protocol. Then, the medium

Table 1 Antibodies used for the western blot experiments

\begin{tabular}{llll}
\hline Antigen & Dilution & Supplier & Charge number \\
\hline LC3 & $1: 1000$ & Sigma & L7543 \\
P62 & 1:1000 & Cell Signaling Technology & 5114 \\
TFEB & 1:1000 & Cell Signaling Technology & 37,785 \\
LAMP1 & $1: 1000$ & Cell Signaling Technology & 9091 \\
Histone H3 & 1:1000 & Sigma & H0164 \\
$\beta$-actin & 1:1000 & Cell Signaling Technology & 4967 \\
\hline
\end{tabular}

Table 2 Sequences of primers used in quantitative RT-PCR

\begin{tabular}{|c|c|c|}
\hline Target gene & Primer & Nucleotide sequence \\
\hline \multirow[t]{2}{*}{ MAP1LC3B } & $\mathrm{F}$ & 5'-AGCAGCATCCAACCAAAATC-3' \\
\hline & $\mathrm{R}$ & 5'-CTGTGTCCGTTCACCAACAG-3' \\
\hline \multirow[t]{2}{*}{ ATP6VOD1 } & $\mathrm{F}$ & 5'-TTCCCGGAGCTITACTTTAACG-3' \\
\hline & $\mathrm{R}$ & 5'-CAAGTCCTCTAGCGTCTCGC-3' \\
\hline \multirow[t]{2}{*}{ UVRAG } & $\mathrm{F}$ & 5'-GGCGTCTTCGACATCTTCGG-3' \\
\hline & $\mathrm{R}$ & 5'-GACGGTCTGGCATAATTCCAAA-3' \\
\hline \multirow[t]{2}{*}{ CTSB } & $\mathrm{F}$ & 5'-GAGCTGGTCAACTATGTCAACA-3' \\
\hline & $\mathrm{R}$ & 5'-GCTCATGTCCACGTTGTAGAAGT-3' \\
\hline \multirow[t]{2}{*}{ LAMP-1 } & $\mathrm{F}$ & 5'-TCTCAGTGAACTACGACACCA-3' \\
\hline & $\mathrm{R}$ & 5'-AGTGTATGTCCTCTTCCAAAAGC-3' \\
\hline \multirow[t]{2}{*}{ TFEB } & $\mathrm{F}$ & 5'-ACCTGTCCGAGACCTATGGG-3' \\
\hline & $\mathrm{R}$ & 5'-CGTCCAGACGCATAATGTTGTC-3' \\
\hline \multirow[t]{2}{*}{$\beta$-actin } & $\mathrm{F}$ & 5'-CGAGGCCCCCCTGAAC-3' \\
\hline & R & 5'-GCCAGAGGCGTACAGGGATA-3' \\
\hline
\end{tabular}

was exchanged with a fresh M199 medium. And the cells were incubated for another $24 \mathrm{~h}$. As indicated, HUVECs were pretreated with $10 \mu \mathrm{M}$ of RSV for $2 \mathrm{~h}$ and then exposed to $200 \mu \mathrm{M}$ of PA for an additional 24h. Subsequently, cells were collected for analysis. All the images were chosen from more than 3 samples and the evaluation process was double-blinded.

\section{ROS generation measurement}

ROS levels were measured by Multi-Mode Microplate Readers (SpectramMax M5) and Confocal microscopy analysis. The determination of intracellular ROS production was measured with the fluorescent probes $2^{\prime}, 7^{\prime}$ dichlorofluorescein diacetate (DCFH-DA). HUVECs were incubated in a 96-well microplate at 8000 cells/well or 16,000 cells/dish. Then cells were dealt with different indicated treatments. Next, HUVECs were treated with $10 \mu \mathrm{M}$ of DCFH-DA at $37^{\circ} \mathrm{C}$ for $20 \mathrm{~min}$, followed by washing with fresh fetal bovine serum-free media three times. The intracellular ROS levels were quantified by a Multi-Mode Microplate Readers or by confocal microscopic analysis (Radiance 2000, Bio-Rad, Hercules, CA). The intensity of emitted fluorescence following DCFHDA treatment was associated with the quantities of ROS in the cell.

\section{Laser scanning confocal microscopy of HUVECs treated with lysotracker red HUVECs were cultured at $16 \times 10^{3}$ cells/dish overnight. Next, cells were treated to the illustrated treatments. Thereafter, cells were washed twice with complete media and incubated with $75 \mathrm{nM}$ lysotracker red for $30 \mathrm{~min}$. Subsequently, cells were washed with PBS three times and were visualized by the confocal microscope}


(Radiance 2000, Bio-Rad, Hercules, CA). Excitation of lysotracker red was at $577 \mathrm{~nm}$ and fluorescence emission was at $590 \mathrm{~nm}$.

\section{MDA assay}

For lipid peroxidation assay, the Lipid Peroxidation MDA assay kit (Beyotime, China) was used to access MDA generation. Based on the manufacturer's procedure, total cell lysate was prepared, and the protein concentration was measured using a BCA assay (Beyotime, China). The MDA levels were quantified by Multi-Mode Microplate Readers (SpectramMax M5) at $532 \mathrm{~nm}$.

\section{Transmission electron microscopy}

HUVECs were harvested and fixed for $2 \mathrm{~h}$, post-fixed with $1 \% \mathrm{OsO}_{4}$ for $1.5 \mathrm{~h}$, washed, and stained in aqueous uranyl acetate for $1 \mathrm{~h}$. The samples were then washed again, dehydrated with graded alcohol, and embedded in Epon-Araldite resin (Canemco \& Marivac, Lakefield, Quebec, Canada). Ultrathin sections were obtained by an ultramicrotome (Reichert-Jung, Inc., Cambridge, UK), counterstained with $0.3 \%$ lead citrate, and visualized on a transmission electron microscope (EM420, Koninklijke Philips Electronics N.V., Amsterdam, The Netherlands).

\section{Statistical analyses}

The statistical analysis was conducted with the t-test and one-way analysis of variance by SPSS 13.0 statistical software (SPSS Inc., IBM, USA). A two-sided $p$-value $<0.05$ was regarded as the statistical significance and the Tukey-Kramer post-hoc test was applied if $p<0.05$. Quantitative data are presented as means \pm standard deviation $(X \pm S D)$. All the experiments were repeated independently at least three times.

\section{Results}

RSV attenuated PA-induced oxidative injury in HUVECs

In accordance with previous observations, HUVECs viability was decreased by PA in a concentration-dependent and time-dependent manner in HUVECs. Cell viability showed $50.38 \%$ decline when treated with at least $200 \mu \mathrm{M}$ of PA than that of $0 \mu \mathrm{M}$ of PA $(p<0.01$, Fig. 1a). After treatment with $200 \mu \mathrm{M}$ of PA for $24 \mathrm{~h}$, the cell
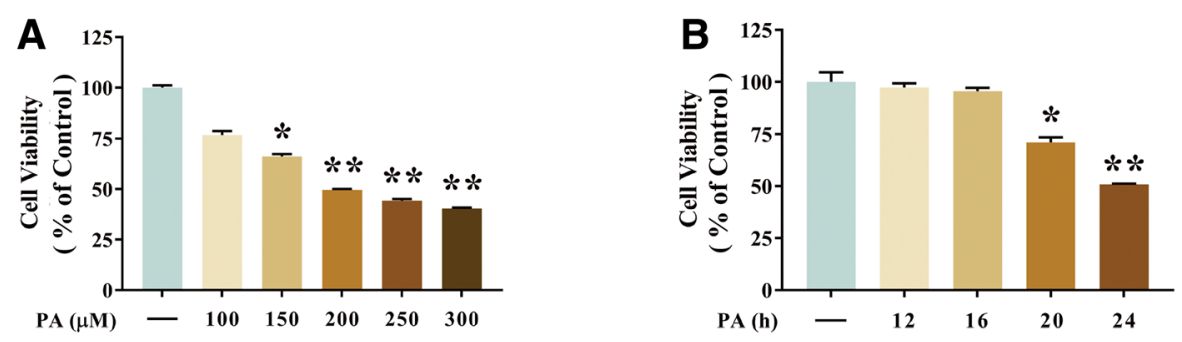

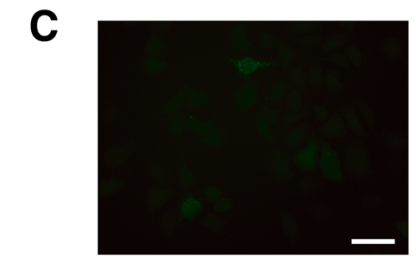

$\mathbf{P A}(\mu \mathrm{M})$

D

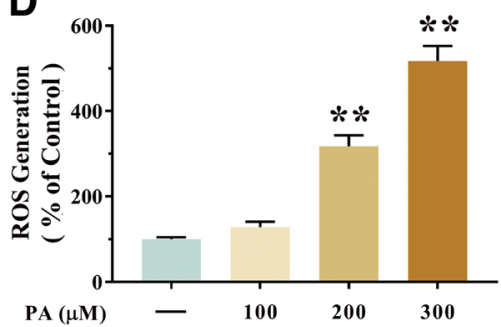

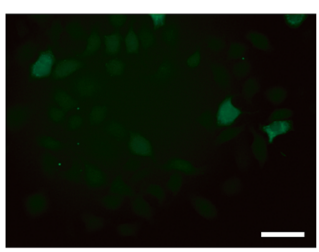

100

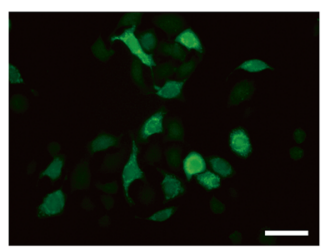

200
E

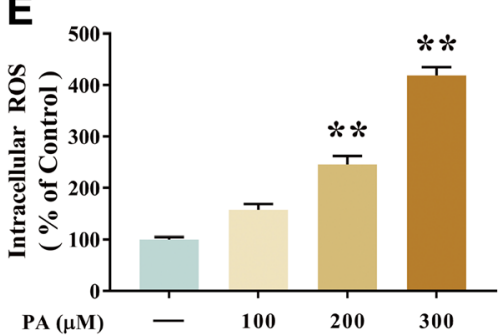

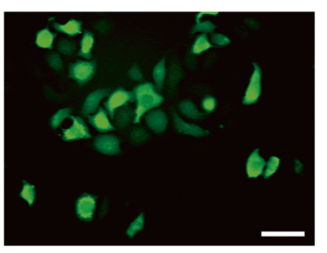

300

$\mathbf{F}$

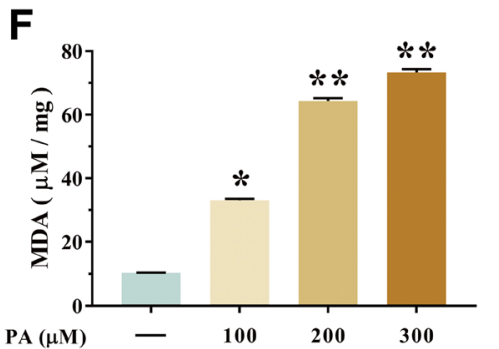

Fig. 1 PA induces oxidative stress in HUVECs. a Cells were incubated with different concentrations (100, 150, 200, 250 and $300 \mu M)$ of PA. Cell viability was measured by CCK-8 detection kit as described in the Materials and methods section. b Cells were incubated with different time (12, 16, 20 and 24 h) of PA. Cell viability was measured by CCK-8 assay. c-e Intracellular ROS levels were estimated using a probe DCFH-DA by confocal microscopy (c) and The bar charts show quantification of the ROS generation levels expressed as the fold change (d), and by microplate reader, fluorescence was read at $485 \mathrm{~nm}$ for excitation and $520 \mathrm{~nm}$ for emission (e). $\mathbf{f}$ The levels of MDA were detected using an MDA detection kit. Values are presented as means $\pm \mathrm{SD}(n=6) ;{ }^{*} p<0.05,{ }^{* *} p<0.01$ compared with control groups 
viability of HUVECs was significantly decreased which was $49.08 \%$ less than that of the initial time point $(p<$ 0.01, Fig. 1b). As shown in Fig. 2a, the protective function of RSV at various concentrations $(0.1,1$ and $10 \mu \mathrm{M})$ was validated by inhibiting PA-induced decreasing cell proliferation in HUVECs. Specially, the precondition of $10 \mu \mathrm{M}$ of RSV for $2 \mathrm{~h}$ could elevate the cell viability significantly which is $58.29 \%$ more than that of the PAtreated HUVECs $(p<0.01$, Fig. 2a). Consistently, intracellular ROS production was the $157.17 \%$ higher than when treated with $200 \mu \mathrm{M}$ of PA than blank group by microplate reader $(p<0.01$, Fig. $2 b)$, which was $212.16 \%$ higher than that of blank group by confocal microscopy $(p<0.01$, Fig. $2 d)$, The MDA level was also $473.44 \%$ elevated by $200 \mu \mathrm{M}$ of PA than that of blank group $(p<0.01$,
Fig. 2e). In contrast, ROS was suppressed by precondition with $10 \mu \mathrm{M}$ of RSV in PA-treated HUVECs, which was $54.29 \%$ less by a microplate reader $(p<0.01$, Fig. $2 \mathrm{~b})$ and $46.88 \%$ less by confocal microscopy than that of PA alone $(p<0.01$, Fig. 2 d). MDA was also significantly decreased by precondition with $10 \mu \mathrm{M}$ of RSV for $2 \mathrm{~h}$, which is $74.35 \%$ less than that of the PA-treated HUVECs $(p<0.01$, Fig. 2e). All these data indicated that RSV pretreatment (especially at a concentration of $10 \mu \mathrm{M})$ for $2 \mathrm{~h}$ attenuated PAinduced injury in HUVECs.

\section{RSV attenuated PA-induced injury through stimulating autophagy in HUVECs}

As shown in Fig. 3a, transmission electron microscopy analysis showed RSV pretreatment increased autophagosomes

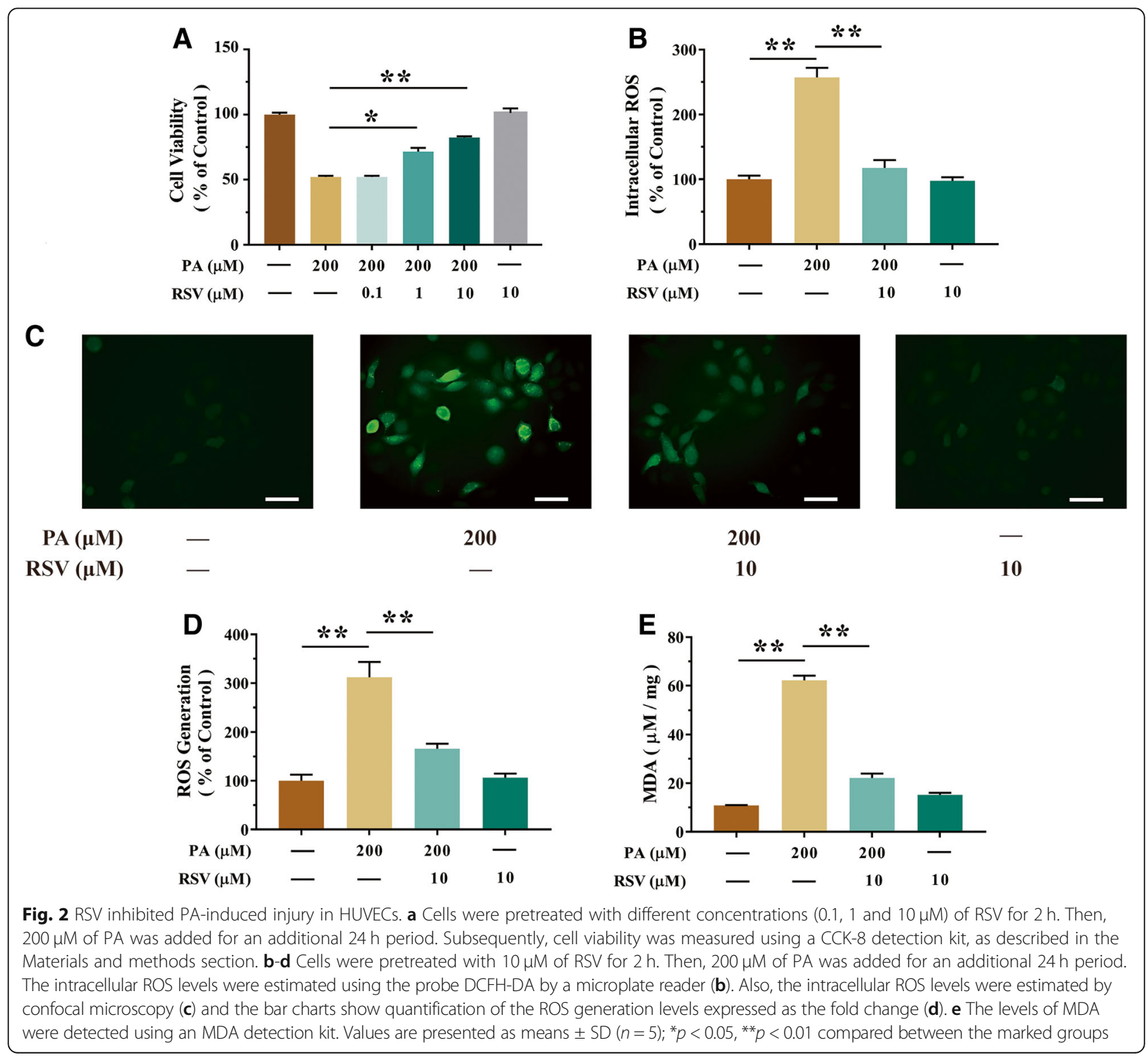




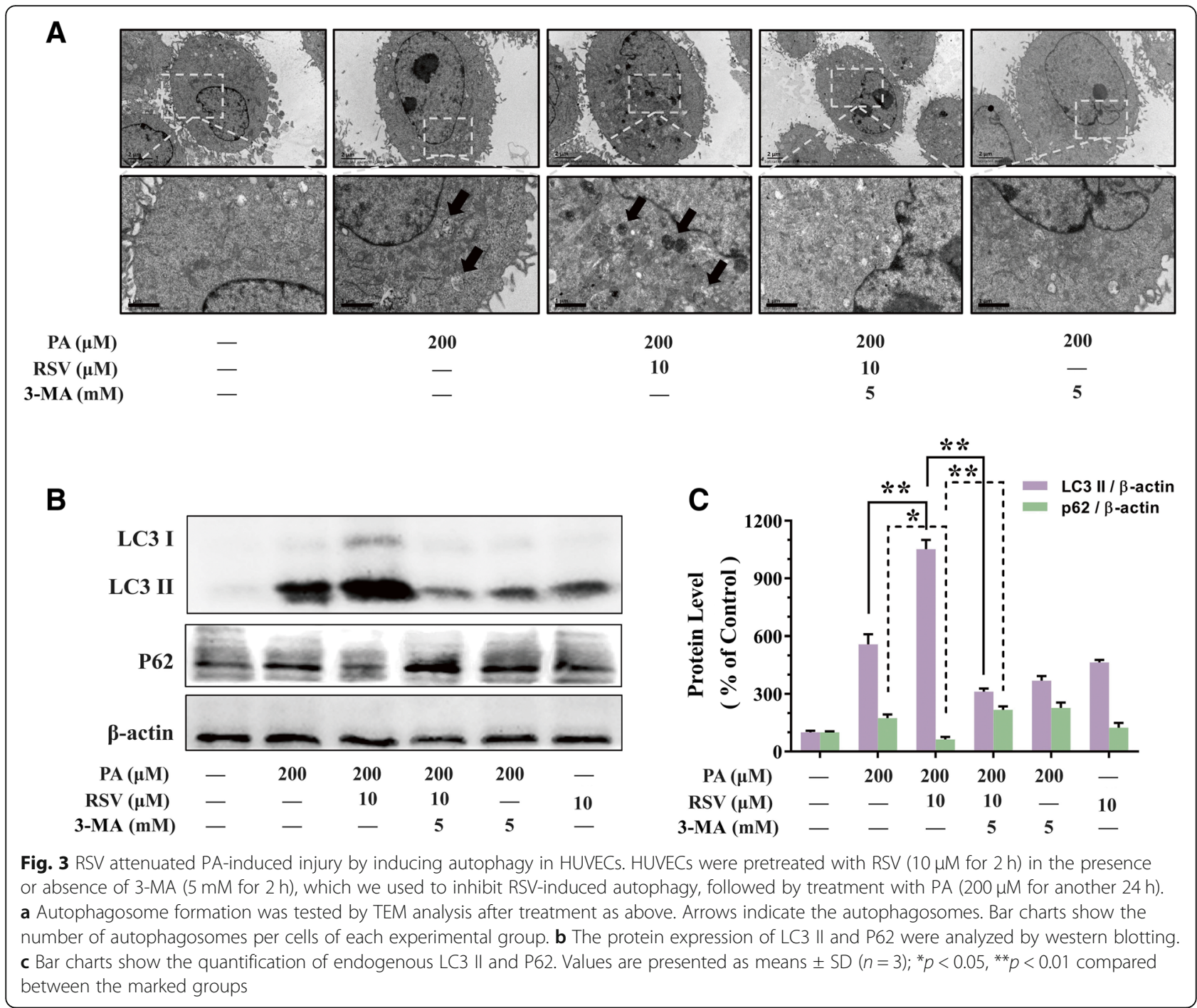

formation $(p<0.05)$. Then, the expression of LC3 II (a precise marker of autophagy) and p62 (a key adaptor protein involved in selective autophagy) expression were analyzed by western blot. As shown in Fig. 3b and c, when treated with PA alone, the expression of LC3 II and P62 were both increased $(p<0.05)$, indicating that autophagic flux was suppressed by PA. However, RSV pretreatment markedly resulted in $88.64 \%$ upregulation of LC3 II expression and 64.43\% downregulation of P62 level in PA-treated HUVECs than that of the group of PA alone $(p<0.05$, Fig. $3 \mathrm{~b}$ and $\mathrm{c}$ ), which indicated that RSV activating autophagy in PA-stimulated HUVECs. Furthermore, pretreatment of 3-MA, an inhibitor of autophagy in the early stage, suppressed RSV-induced autophagosome formation (Fig. 3a), as well as, suppressed the increased expression of LC3-II and decreasing expression of P62 $(p<0.01$, Fig. $3 \mathrm{~b}$ and $\mathrm{c})$. Consequently, these data indicated that RSV attenuated endothelial oxidative injury in an autophagy-dependent manner.
RSV increases TFEB activity in HUVECs

TFEB is an important transcription factor of the MiT/ TFE family that regulates lysosomal biogenesis and autophagy [21]. Many downstream genes of TFEB, including ATP6V0D1, LAMP1, CTSB, MAP 1LC3B and UVRAG, are involved in lysosome-associated processes and substrate degradation [22]. The activation of TFEB could be examined based on that its active form is located in cell nuclei while its inactive form is in the cytoplasm. As shown in Fig. 4 a and b, the protein expression of nucleus-TFEB and cytoplasm-TFEB induced by RSV with different concentrations $(0.1,1$ and $10 \mu \mathrm{M})$ were analyzed by western blotting. We found that RSV exposure could activate TFEB, especially elevated the nucleus-TFEB expression. $10 \mu \mathrm{M}$ of RSV markedly resulted in the $174.54 \%$ upregulation the protein expression of nucleus-TFEB and $128.19 \%$ upregulation of cytoplasm-TFEB than that of the blank group $(p<0.01$, Fig. 4b) in HUVECs. Meanwhile, the ratio of the 


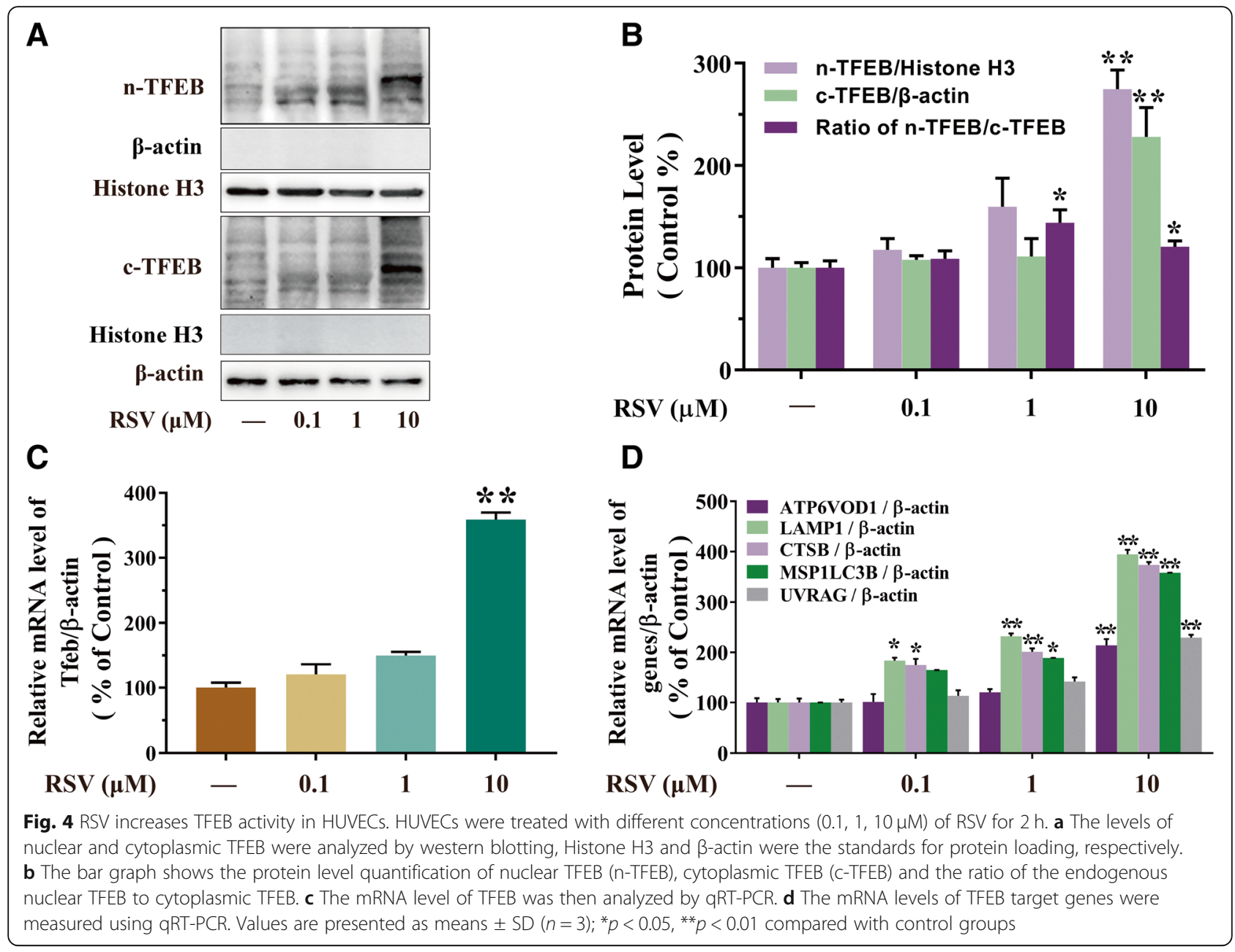

nucleus- to cytoplasm-TFEB was $20.3 \%$ elevated by $10 \mu \mathrm{M}$ of RSV than that of the blank group ( $p<0.05$, Fig. $4 \mathrm{~b})$ in HUVECs. Furthermore, we examined the mRNA expression of TFEB, which presented comparable results in Fig. 4c. The mRNA expression of TFEB was $258.96 \%$ upregulated by the treatment of RSV than that of the blank group $(p<0.01$, Fig. 4c). Subsequently, our study showed $10 \mu \mathrm{M}$ of RSV increased the nucleus translocation of TFEB along with an increased expression of TFEBresponsive genes. As we predicted, RSV increased the mRNA expression of ATP6V0D1, LAMP1, CTSB, MAP 1LC3B and UVRAG significantly (Fig. 4d). In general, these data shown that RSV motivated TFEB to translocate into the nucleus, elevated TFEB expression and activating TFEB-downstream genes related to autophagy and lysosomal biosynthesis.

RSV-induced autophagy in PA-stimulated endothelial cells in a TFEB-dependent manner

To further examine the capability of TFEB in RSVinduced autophagy, TFEB-specific siRNA (siTFEB) was transfected as described in the Materials and methods section, TFEB mRNA expression was significantly upregulated by RSV in PA-stimulated HUVECs, while it was knocked-down by TFEB siRNA transfection (Fig. 5a). The mRNA expression of TFEB was $248.55 \%$ upregulated by the RSV precondition in the PA-treated HUVECs than that of the group of PA alone $(p<0.01$, Fig. 5a). However, after treatment of TFEB siRNA, it resulted in $57.32 \%$ downregulation of $\mathrm{n}$-TFEB $(p<0.01$, Fig. $5 \mathrm{c}$ ) and in $11.05 \%$ downregulation of the ratio of $n-$ TFEB to c-TFEB $(p<0.05$, Fig. $5 c)$ in the PA-treated HUVECs than that of the group of RSV precondition. Meanwhile, the expression of RSV-induced upregulate of nuclear-TFEB, cytoplasmic-TFEB, LAMP1 and downregulate of P62 were significantly inhibited by siTFEB transfection in PA-treated HUEVCs (Fig. 5b-e). Furthermore, RSV-induced gene expression of lysosomal biosynthesis and autophagy was decreased by suppression of TFEB (Fig. 5f). In addition, confocal microscopy analysis exhibited that RSV exposure increased lysosomes in PA-treated HUVECs. And the function of RSV was $76.25 \%$ downregulated by SITFEB in PA-treated HUVECs than that of the group of RSV precondition ( $p<0.01$, Fig. $5 \mathrm{~g}$ and $\mathrm{h})$. 


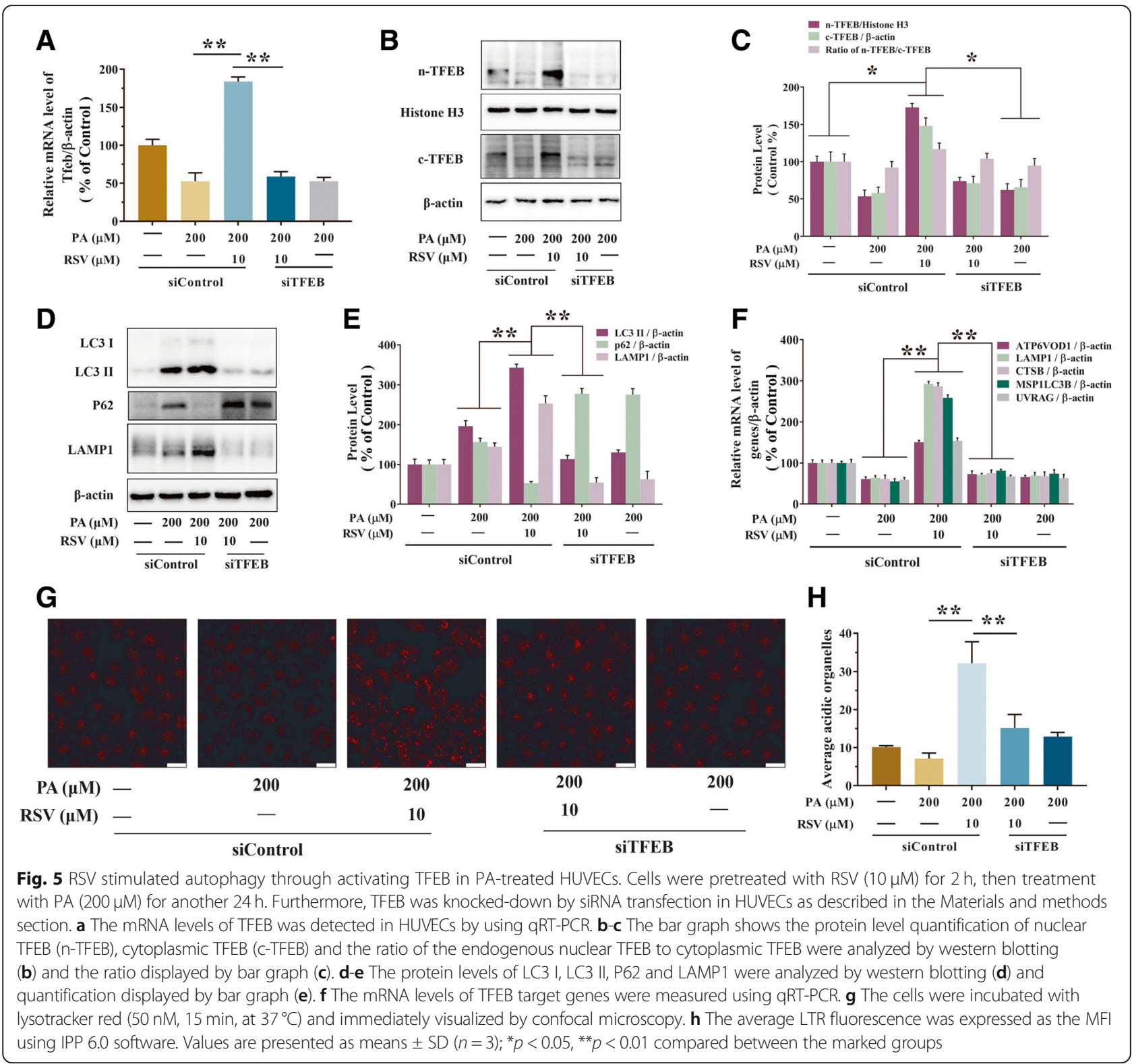

Further, the protective function of RSV in high levels of ROS and MDA caused by PA was also inhibited by siTFEB (Fig. 6a-d). As well, the ROS generation was significantly elevated by $S i T F E B$ in PA-treated HUVECs, which is $52.83 \%$ more by confocal microscopy $(p<0.01$, Fig. 6 a and b) and $76.51 \%$ more by a microplate reader than the group of RSV precondition ( $p<0.01$, Fig. 6c). Meanwhile, the CCK- 8 analysis found that RSV resulted in the $29.93 \%$ upregulation of cell viability when inhibited by SITFEB in PA-treated HUEVCs than that of the group of RSV precondition ( $p<0.01$, Fig. 6e). These results demonstrated that RSV reduced PA-induced oxidative stress by activating the TFEB-dependent autophagy pathway.

\section{Discussion}

In this study, we provide evidence, for the first time, that RSV attenuates endothelial oxidative injury in HUVECs by inducing autophagy, partly by activating the TFEB signaling pathway. Specifically, we observed that (1) RSV can suppress oxidative stress and increase the viability of endothelial cells via autophagy induction and (2) the protective autophagy induced by RSV in HUVECs depends on a TFEB pathway.

Oxidative stress is regarded as the main inducer of endothelial dysfunction, which is the initial and crucial pathological process of AS [23]. In fact, all risk factors for cardiovascular diseases, such as high blood pressure, diabetes, and smoking, as well as the cardiovascular 

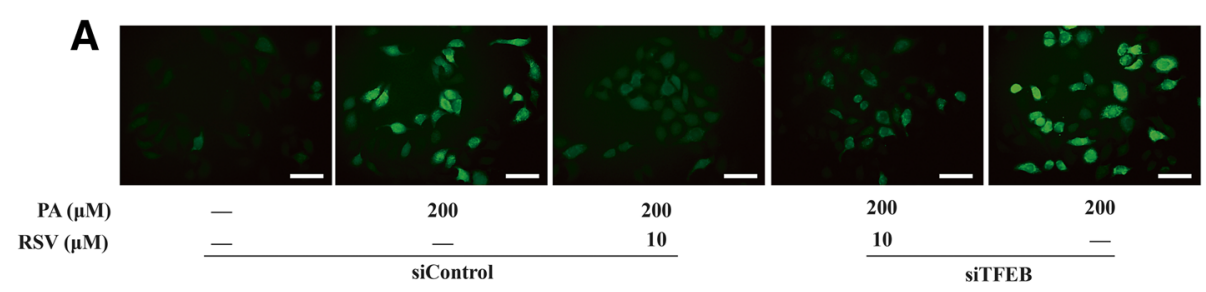

B

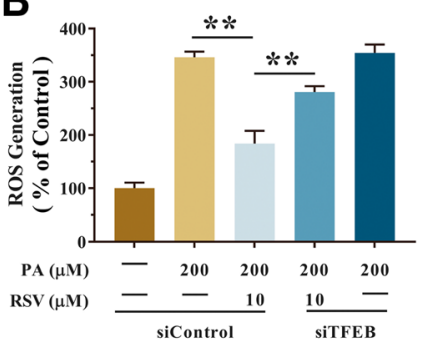

D

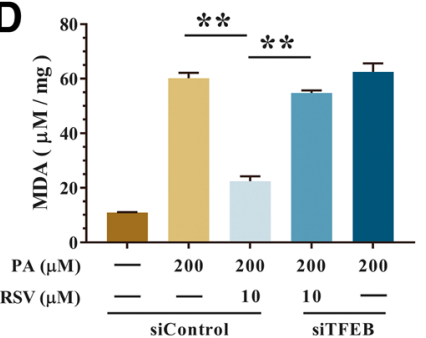

C

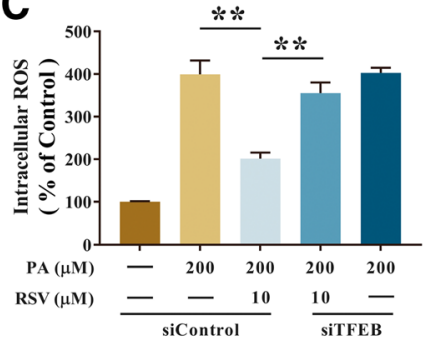

$\mathbf{E}$

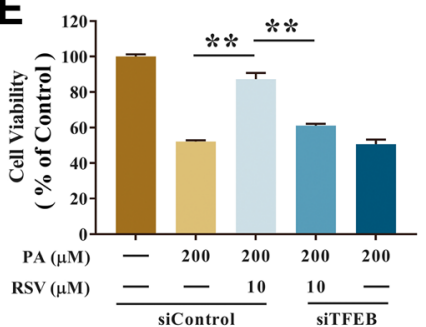

Fig. 6 RSV attenuated oxidative injury through stimulating autophagy in a TFEB-dependent manner in PA-treated HUVECs. Cells were pretreated with RSV $(10 \mu \mathrm{M})$ for $2 \mathrm{~h}$, then treatment with PA $(200 \mu \mathrm{M})$ for another $24 \mathrm{~h}$. Furthermore, TFEB was knocked-down by siRNA transfection in HUVECS as described in the Materials and methods section. a-c Intracellular ROS levels were estimated using a probe DCFH-DA by confocal microscopy (a) and bar charts show quantification of the ROS generation levels expressed as the fold change (b), and by microplate reader, fluorescence was read at 485 $\mathrm{nm}$ for excitation and $520 \mathrm{~nm}$ for emission (c). $\mathbf{d}$ The levels of MDA were detected using an MDA detection kit. e The cell viability was measured using a CCK-8 detection kit, Values are presented as means \pm SD $(n=6) ;{ }^{*} p<0.05,{ }^{* *} p<0.01$ compared between the marked groups

disease itself, are associated with increased ROS production in the vessel wall, which ultimately leads to oxidative stress $[24,25]$. In this study, an endothelial oxidative stress model was established with PA, which is the main saturated free fatty acid in the bloodstream. Previously, PA is widely used to establish injury model in many metabolic diseases, including insulin resistance [26] and oxidative stress [27]. Particularly, PA has been used to treat ECs to induce endothelial oxidative stress as previously reported, which were consistent with our results. For example, PA reportedly induces oxidative injury and causes endothelial dysfunction. Jiang $\mathrm{H}$ et al. found that PA promotes the apoptosis of endothelial progenitor cells via p38 and JNK mitogen-activated protein kinase pathways [6]. Staiger et al. found that PA can induce vascular inflammation by increasing IL-6 production in human coronary artery endothelial cells [28]. In addition, Cacicedo et al. found that PA can induce apoptosis in cultured bovine retinal pericytes by increasing oxidative stress [29]. In contrast, the model of insulin resistance induced by PA was generally reported in the studies of the skeletal muscle system. Thus, we consider endothelial cells exposed to PA represent a suitable model for exploring the anti-oxidative mechanism of RSV.

Several pharmacological treatments for AS have been proposed, but few have exhibited both efficacy and longterm safety. Several pieces of evidence indicate that phytochemicals, which are abundant and available in the daily diet, can protect the cardiovascular system against AS by alleviating endothelial oxidative injury [30]. Specifically, the ameliorative effects of RSV on ASassociated oxidative stress have been confirmed in several studies [31-33]. Furthermore, RSV can protect endothelial cells against AS by decreasing ROS production, increasing NO production, and increasing the production of inflammatory factors [34, 35]. Therefore, we believe that the protective effects against AS are mainly related to the antioxidant activities of RSV. Due to the low bioavailability of RSV in vivo, the serum concentration of unmetabolized RSV generally peak in the sub- to the low-micromolar range and the peak concentration 
reached in the first $30 \mathrm{~min} \sim 2 \mathrm{~h}$. In our previous study, we found resveratrol was tolerated by endothelial cells which exhibit no significant negative effect on cell viability up to the concentration of $10 \mu \mathrm{M}$. Accordingly, we chose a series of concentrations $(0.1,1$ and $10 \mu \mathrm{M})$ of RSV to investigate the effect on autophagy of endothelial cells. We hope to find whether RSV can affect autophagy in endothelial cells at the concentrations that cells showed no changes in cell viability. Moreover, the concentrations applied in our study were in line with some previously published works $[8,36]$. Autophagy is a cellular housekeeping process involved in the turnover of cytoplasmic organelles and proteins through a lysosomedependent degradation process, and it plays a critical role in cancers, neurodegenerative disorders, and cardiovascular diseases [37-39]. Autophagy has been described as a major cytoprotective mechanism associated with phytochemicals [40]. Additionally, RSV may attenuate endothelial inflammation by inducing autophagy [20]. Moreover, the neuroprotective effects of RSV against Parkinson's disease and Alzheimer's disease are due to autophagy [41, 42]. These findings strongly indicate that autophagy is critical for the multiple RSV-induced bioprotective effects. Thus, we examined the role of autophagy in RSV-induced endothelial protection in PAstimulated HUVECs. In this study, the RSV pretreatment significantly ameliorated endothelial oxidative injury in PA-treated HUVECs, as indicated by increasing cell proliferation and decreasing ROS and MDA levels (Fig. 2). Furthermore, PA-induced oxidative injury increased the production of LC3 II and P62, implying that autophagic flux was blocked. The RSV pretreatment considerably increased and decreased the LC3 II and P62 levels, respectively, which demonstrated that RSV induces autophagic flux. However, a 3-MA treatment attenuated the RSV-induced formation of autophagosomes (Fig. 3). These results suggested that an autophagydependent mechanism is important for the RSV-mediated attenuation of oxidative injury in PA-stimulated HUVECs. However, the exact mechanisms underlying RSV-induced autophagy remain uncharacterized.

Settembre et al. recently reported that TFEB is a key regulator of an autophagy-lysosome signaling pathway in a starvation model. TFEB is also required to induce the expression of starvation-response genes involved in several lipid catabolism steps occurring in different cellular compartments, such as the transport of fatty acid chains and the oxidation of free fatty acids in mitochondria and peroxisomes. Previous study revealed that the TFEB overexpression may affect lipid catabolismm, inhibit etabolic activities, and subsequently ameliorate the obesity-related metabolic syndrome [16]. Thus, activating TFEB may extend the lifespan of $C$. elegans, indicating this transcription factor influences longevity [12].
We previously observed that RSV can promote longevity [43]. Therefore, we presumed that there is a relationship between RSV and TFEB. There are reports that TFEB has a global transcriptional regulatory function during lipid catabolism because it directly regulates the proliferator-activated receptor gamma coactivator- $1 \alpha$ $(\mathrm{PGC} 1 \alpha)$. Moreover, RSV may prevent renal lipotoxicity through the AMPK-SIRT1-PGC1 $\alpha$ axis [44]. Furthermore, TFEB activity is regulated transcriptionally and post-transcriptionally by nutrients [16]. Therefore, we hypothesized that TFEB may be regulated by RSV. In this study, we aimed to verify whether TFEB is necessary for the RSV-induced autophagy that protects PA-treated HUVECs against oxidative injury. Previous studies have shown the activation of TFEB could be reflected by the total protein expression of the total nuclear TFEB and the ratio of nuclear TFEB/ cytosolic TFEB by western blot comprehensively [45-48]. In our study, we found $1 \mu \mathrm{M}$ of RSV could increase the ratio of nuclear TFEB/ cytosolic TFEB but not elevate the total nuclear TFEB. However, $10 \mu \mathrm{M}$ of RSV could significantly increase the ratio of nuclear TFEB/ cytosolic TFEB but not elevate the total nuclear TFEB. Thus, we argue that the $10 \mu \mathrm{M}$ of RSV could trigger the nuclear translocation of TFEB, namely activating the TFEB in the endothelial cells. We observed that TFEB expression in HUVECs may be upregulated by RSV in a concentration-dependent manner, with $10 \mu \mathrm{M}$ of RSV resulting in the most efficient TFEB expression. We also examined the expression of the downstream targets of TFEB, including MAP $1 L C 3 B$ and $U V R A G$, which encode proteins affecting autophagy progression, and ATP6VOD1, LAMP1 and CTSB, which encode lysosomal transmembrane proteins and lysosomal hydrolase. In addition to regulating TFEB production, RSV also induced a concentration-dependent increase in the expression of these genes in HUVECs (Fig. 4). These results indicate that TFEB expression is positively regulated by RSV, implying that TFEB represents a novel RSV target related to the protection against endothelial oxidative damage. As expected, the RSV-induced autophagy process and endothelial protective effect were notably attenuated when PA-treated HUVECs were transfected with TFEB siRNA (Figs. 5 and 6). This confirms that TFEB is indispensable for the RSV-induced autophagy in the endothelial oxidative injury model.

There are still some limitations in this study, which require further explanation. First, our data were based on isolated HUVECs in vitro, but not the more complex microenvironment in vivo. Whether RSV stimulates autophagy via activating TFEB in vivo need to be validated further. Besides, it also needs to be explored whether there is the other mechanism of anti-AS effects of RSV and which one is dominant in a different situation. This finding provides valuable clues regarding the potential 


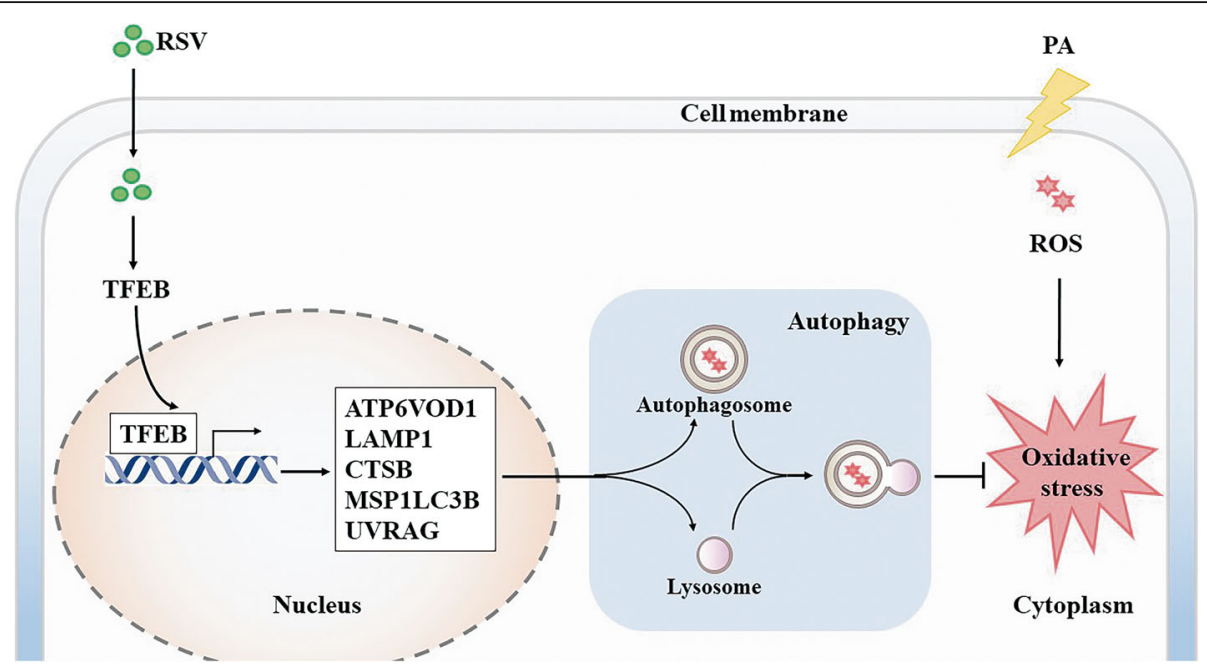

Fig. 7 Schema of RSV-induced autophagy via activating TFEB in PA-stimulated HUVECs. Depending on the proposed signaling pathways: RSV activates TFEB and TFEB-target genes. Further, inducing the entire autophagy progress, concluding autophagosome as well as lysosome formation, ultimately, RSV attenuates PA-caused endothelial oxidative injury

protective role of RSV. We provided critical experimental evidence of the good prospect of RSV in the clinical treatment of cardiovascular diseases. It opened a view in the search of new mitochondria-targeting drugs for anti-AS therapy.

\section{Conclusion}

To the best of our knowledge, the data presented here reveals for the first time that RSV protects HUVECs against oxidative injury caused by PA via autophagy mediated by a TFEB-dependent mechanism (Fig. 7). Our results provide new insights into the possibility that TFEB is targeted by RSV, at least partially, and suggest that the associated induced autophagy may have implications for improving cardiovascular health. These results provide critical experimental evidence of the potential utility of the clinical application of RSV for preventing and treating AS.

\section{Abbreviations}

3-MA: 3-methyladenine; AS: Atherosclerosis; ATP6V0D1: ATPase H+ Transporting V0 Subunit D1; CTSB: Cathepsin B; HUVECS: Human umbilical vein endothelial cells; LAMP-1: Lysosomal-associated membrane protein 1; MAP1LC3B: Microtubule-associated proteins 1A/1B light chain 3B; PA: Palmitic acid;ROS, reactive oxygen species; siRNA: Small interfering RNA; TFEB: Transcription factor EB; UVRAG: UV resistance-associated gene

\section{Acknowledgments}

We would also like to thank the technicians in Biological Testing Center of Third Military Medical University for their assistance in experiments.

\section{Authors' contributions}

$M M T, Y L$ and $X Z$ designed the study; $X Z$, JY, MZ and $Y Z$ conducted the experiments; $Y L, P H$ and $X L$ helped in interpreting data. $X Z$ did sample analysis and data analysis, $X Z$ wrote the manuscript; MMT and $Y L$ revised the paper. All authors read and approved the final manuscript.

\section{Funding}

This work was supported by the National Natural Science Foundation of China (grant number:81502804).

\section{Availability of data and materials}

Access to the data of this study will be considered by the corresponding author upon reasonable request.

Ethics approval and consent to participate

The experiments were supervised by the Ethics Committee of Third Military Medical University [SYXK (YU) 2012-0012].

\section{Consent for publication}

Not applicable.

\section{Competing interests}

The authors declare that they have no competing interests.

Received: 22 March 2019 Accepted: 25 June 2019

Published online: 02 July 2019

\section{References}

1. Chang P, Wruck L, Shahar E, Rossi J, Loehr L, Russell S, Agarwal S, Konety S, Rodriguez C, Rosamond W. Trends in hospitalizations and survival of acute decompensated heart failure in four US communities (2005-2014): the atherosclerosis risk in communities (ARIC) study community surveillance. Circulation. 2018;138(1):12-24.

2. Shaw LJ, Goyal A, Mehta C, Xie J, Phillips L, Kelkar A, Knapper J, Berman DS, Nasir K, Veledar E. 10-year resource utilization and Costsfor cardiovascular care. J Am Coll Cardiol. 2018;71:1078-89.

3. Schober A, Weber C. Mechanisms of MicroRNAs in atherosclerosis. Annu Rev Pathol. 2016;11:583.

4. Tajbakhsh A, Rezaee M, Kovanen PT, Sahebkar A. Efferocytosis in atherosclerotic lesions: malfunctioning regulatory pathways and control mechanisms. Pharmacol Ther. 2018:188:12-25.

5. Chen P, Liu H, Xiang H, Zhou J, Zeng Z, Chen R, Zhao S, Xiao J, Shu Z, Chen $\mathrm{S}, \mathrm{Lu} \mathrm{H}$. Palmitic acid-induced autophagy increases reactive oxygen species via the $\mathrm{Ca} 2+/ \mathrm{PKCa} / \mathrm{NOX} 4$ pathway and impairs endothelial function in human umbilical vein endothelial cells. Exp Ther Med. 2019;17(4):2425-32.

6. Jiang H, Liang C, Liu X, Jiang Q, He Z, Wu J, Pan X, Ren Y, Fan M, Li MJA. Palmitic acid promotes endothelial progenitor cells apoptosis via p38 and JNK mitogen-activated protein kinase pathways. Atherosclerosis. 2010;210:71-7. 
7. Kopp P. Resveratrol, a phytoestrogen found in red wine. A possible explanation for the conundrum of the'French paradox. Eur J Endocrinol. 1998;138(6):619-20.

8. Zhou X, Chen M, Zeng X, Yang J, Deng H, Yi L, Mi M. Resveratrol regulates mitochondrial reactive oxygen species homeostasis through Sirt3 signaling pathway in human vascular endothelial cells. Cell Death Dis. 2014;5:e1576.

9. Kraft C, Martens S. Mechanisms and regulation of autophagosome formation. Curr Opin Cell Biol. 2012;24:496-501.

10. Rubinsztein DC, Mariño G, Kroemer G. Autophagy and aging. Cell. 2011;146: 682-95.

11. Mizushima N, Levine B, Cuervo AM, Klionsky DJ. Autophagy fights disease through cellular self-digestion. Nature. 2008;451:1069.

12. Bravo-San Pedro JM, Kroemer G, Galluzzi L. Autophagy and Mitophagy in cardiovascular disease. Circ Res. 2017;120:1812.

13. Levine B, Kroemer G. Autophagy in the pathogenesis of disease. Cell. 2008; 132:27-42.

14. Wang $B$, Yang $Q$, Sun $Y Y$, Xing $Y F$, Wang $Y B$, Lu XT, Bai WW, Liu XQ, Zhao YX. Resveratrol-enhanced autophagic flux ameliorates myocardial oxidative stress injury in diabetic mice. J Cell Mol Med. 2014;18:1599-611.

15. Li L, Hai J, Li Z, Zhang Y, Peng H, Li K, Weng X. Resveratrol modulates autophagy and NF-kB activity in a murine model for treating non-alcoholic fatty liver disease. Food Chem Toxicol. 2014;63:166-73.

16. Settembre C, Cegli RD, Mansueto G, Saha PK, Vetrini F, Visvikis O, Huynh T, Carissimo A, Palmer D, Klisch TJ. TFEB controls cellular lipid metabolism through a starvation-induced autoregulatory loop. Nat Cell Biol. 2013;15:647.

17. Settembre C, Di MC, Polito VA, Garcia AM, Vetrini F, Erdin S, Erdin SU, Huynh T, Medina D, Colella P. TFEB links autophagy to lysosomal biogenesis. Science. 2011;332:1429-33.

18. Sardiello M, Palmieri M, Ronza AD, Medina DL, Valenza M, Gennarino VA Malta CD, Donaudy F, Embrione V, Polishchuk RS. A gene network regulating lysosomal biogenesis and function. Science. 2009;325:473.

19. Wood JG, Rogina B, Lavu S, Howitz K, Helfand SL, Tatar M, Sinclair DJN. Sirtuin activators mimic caloric restriction and delay ageing in metazoans. Nature. 2004;430:686

20. Chen ML, Yi L, Jin X, Liang XY, Zhou Y, Zhang T, Xie Q, Zhou X, Chang H, Fu YJ. Resveratrol attenuates vascular endothelial inflammation by inducing autophagy through the CAMP signaling pathway. Autophagy. 2013;9: 2033-45.

21. Napolitano G, Ballabio A. TFEB at a glance. J Cell Sci. 2016;129:2475.

22. Saftig P, Haas A. Turn up the lysosome. Nat Cell Biol. 2016;18:1025-7.

23. Li H, Horke S, Förstermann UJA. Vascular oxidative stress, nitric oxide and atherosclerosis. Atherosclerosis. 2014;237:208-19.

24. Stocker R, Keaney JFJPR. Role of oxidative modifications in atherosclerosis. Physiol Rev. 2004;84:1381-478.

25. Harrison D, Griendling KK, Landmesser U, Hornig B, HJTAjoc D. Role of oxidative stress in atherosclerosis. Am J Cardiol. 2003;91:7-11.

26. Alkhateeb $\mathrm{H}$, Qnais E. Preventive effect of oleate on palmitate-induced insulin resistance in skeletal muscle and its mechanism of action. J Physiol Biochem. 2017:73:605-12

27. Lee C-H, Lee S-D, Ou H-C, Lai S-C, Cheng Y-J. Eicosapentaenoic acid protects against palmitic acid-induced endothelial dysfunction via activation of the AMPKJeNOS pathway. Int J Mol Sci. 2014;15:10334-49.

28. Staiger H, Staiger K, Stefan N, Wahl HG, Machicao F, Kellerer M, Häring HUJD. Palmitate-induced interleukin-6 expression in human coronary artery endothelial cells. Diabetes. 2004;53:3209.

29. Cacicedo JM, Benjachareowong SE, Ruderman NB, Ido YJD. Palmitateinduced apoptosis in cultured bovine retinal Pericytes: roles of NAD(P) $H$ oxidase, oxidant stress, and ceramide. Diabetes. 2005;54:1838-45.

30. Jin X, Chen M, Yi L, Chang H, Zhang T, Wang L, Ma W, Peng X, Zhou Y, Mi M. Delphinidin-3-glucoside protects human umbilical vein endothelial cells against oxidized low-density lipoprotein-induced injury by autophagy upregulation via the AMPK/SIRT1 signaling pathway. Mol Nutr Food Res. 2014:58:1941-51.

31. Pollack RM, Barzilai N, Anghel V, Kulkarni AS, Golden A, O'Broin P, Sinclair DA, Bonkowski MS, Coleville AJ, Powell D: Resveratrol improves vascular function and mitochondrial number but not glucose metabolism in older adults. J Gerontol A Biol Sci Med Sci 2017;pii:3073145.

32. Li YR, Li S, Lin CC. Effect of resveratrol and pterostilbene on aging and longevity. Biofactors. 2017.

33. Bonkowski MS, Sinclair DA. Slowing ageing by design: the rise of NAD+ and sirtuin-activating compounds. Nat Rev Mol Cell Biol. 2016;17:679-90.
34. Petrovski G, Gurusamy N, Das DK. Resveratrol in cardiovascular health and disease. Ann N Y Acad Sci. 2011;1215:22-33.

35. Csiszar A. Anti-inflammatory effects of resveratrol: possible role in prevention of age-related cardiovascular disease. Ann N Y Acad Sci. 2011; 1215:117-22.

36. Yang J, Zhou X, Zeng X, Hu O, Yi L, Mi MJN, Metabolism: Resveratrol attenuates oxidative injury in human umbilical vein endothelial cells through regulating mitochondrial fusion via TyrRS-PARP1 pathway. 2019;16:9.

37. Yang J, Zhou X, Zeng X, Ou HU, Long YI, Mantian MI. Resveratrol attenuates oxidative injury in human umbilical vein endothelial cells through regulating mitochondrial fusion via TyrRS-PARP1 pathway. Nutr Metab (Lond). 2019;16:9.

38. Carew JS, Kelly KR, Nawrocki ST. Autophagy as a target for cancer therapy: new developments. Cancer Manage Res. 2012;4:357.

39. Irrcher I, Park DS. Parkinson's disease: to live or die by autophagy. Sci Signal. 2009;2:pe21.

40. Delmas D, Solary E. Latruffe NJCmc: resveratrol, a phytochemical inducer of multiple cell death pathways: apoptosis, autophagy and mitotic catastrophe. Curr Med Chem. 2011;18:1100-21.

41. Wu Y, Li X, Zhu JX, Xie W, Le W, Fan Z, Jankovic J, Pan T. Resveratrolactivated AMPK/RIRT1/autophagy in cellular models of Parkinson's disease. Neurosignals. 2011:19:163-74.

42. Deng H. Mi M-tJNr: resveratrol attenuates A $25-35$ caused neurotoxicity by inducing autophagy through the TYrRS-PARP1-SIRT1 signaling pathway. Neurochem Res. 2016;41:2367-79.

43. Morselli E, Maiuri MC, Markaki M, Megalou E, Pasparaki A, Palikaras K, Criollo A, Galluzzi L, Malik SA, Vitale I, Michaud M, Madeo F, Tavernarakis N, Kroemer G. Caloric restriction and resveratrol promote longevity through the Sirtuin-1dependent induction of autophagy. Cell Death Dis. 2010;1:e10-188.

44. Kim MY, Lim JH, Youn HH, Hong YA, Yang KS, Park HS, Chung S, Ko SH, Shin SJ, Choi BS. Resveratrol prevents renal lipotoxicity and inhibits mesangial cell glucotoxicity in a manner dependent on the AMPK-SIRT1-PGC1a axis in db/db mice. Diabetologia. 2013:56:204-17.

45. Ye B, Wang Q, Hu H, Shen Y, Fan C, Chen P, Ma Y, Wu H, Xiang MJA. Restoring autophagic flux attenuates cochlear spiral ganglion neuron degeneration by promoting TFEB nuclear translocation via inhibiting MTOR. Autophagy. 2019:1-19.

46. Ma X, Mani K, Liu H, Kovacs A, Murphy JT, Foroughi L, French BA, Weinheimer CJ, Kraja A, Benjamin IJJJAHA. Transcription factor EB activation rescues advanced aB-crystallin mutation-induced cardiomyopathy by normalizing desmin localization. J Am Heart Assoc. 2019;8:e010866.

47. Zheng G, Pan Z, Zhan Y, Tang Q, Zheng F, Zhou Y, Wu Y, Zhou Y, Chen D, Chen JJO. Cartilage: TFEB protects nucleus pulposus cells against apoptosis and senescence via restoring autophagic flux. Osteoarthr Cartil. 2019;27: 347-57

48. Magini A, Polchi A, Di Meo D, Mariucci G, Sagini K, De Marco F, Cassano T, Giovagnoli S, Dolcetta D, CJHmg E. TFEB activation restores migration ability to Tsc1-deficient adult neural stem/progenitor cells. Hum Mol Genet. 2017; 26:3303-12.

\section{Publisher's Note}

Springer Nature remains neutral with regard to jurisdictional claims in published maps and institutional affiliations.

Ready to submit your research? Choose BMC and benefit from

- fast, convenient online submission

- thorough peer review by experienced researchers in your field

- rapid publication on acceptance

- support for research data, including large and complex data types

- gold Open Access which fosters wider collaboration and increased citations

- maximum visibility for your research: over $100 \mathrm{M}$ website views per year

At BMC, research is always in progress.

Learn more biomedcentral.com/submission 